\title{
Recurrent Malignant Thyroid Gland Neoplasm
}

National Cancer Institute

\section{Source}

National Cancer Institute. Recurrent Malignant Thyroid Gland Neoplasm. NCI Thesaurus.

Code C150538.

Reemergence of a malignant thyroid gland neoplasm after a period of remission. 